\title{
eJRIEPS
}

Ejournal de la recherche sur l'intervention en éducation physique et sport

Hors-série $\mathrm{N}^{\circ} 2 \mid 2018$

À propos de certaines bases théoriques et pratiques des sports collectifs

\section{Le collectif, le groupe, l'équipe : une histoire de temps... mais pas seulement}

Jean-Francis Gréhaigne

\section{OpenEdition}

Journals

Édition électronique

URL : http://journals.openedition.org/ejrieps/508

DOI : 10.4000/ejrieps.508

ISSN : 2105-0821

Éditeur

ELLIADD

Référence électronique

Jean-Francis Gréhaigne, « Le collectif, le groupe, l'équipe : une histoire de temps... mais pas seulement », eJRIEPS [En ligne], Hors-série № 2 | 2018, mis en ligne le 01 juillet 2018, consulté le 10 décembre 2020. URL : http://journals.openedition.org/ejrieps/508 ; DOI : https://doi.org/10.4000/ ejrieps.508

La revue eJRIEPS est mise à disposition selon les termes de la Creative Commons Attribution 4.0 International License. 


\section{Le collectif, le groupe, l'équipe : une histoire de temps ... mais pas seulement}

Institutionnellement, la pratique des jeux collectifs est habituellement l'occasion d'une adhésion à une association : on est membre d'une équipe, on appartient à une communauté, on joue avec des partenaires contre des adversaires... Un collectif de joueurs dans les sports d'équipe ne vise pas toujours la recherche à tout prix de la performance. Il peut être couramment un prétexte à la connaissance de soi, l'occasion de constructions collectives et de partages, de plaisir du jouer ensemble contre les autres, une belle circulation de la balle, le tout renvoyant à une production collective du groupe. Employés l'un pour l'autre, « groupe » «équipe » et « collectif » ne sont pas toujours définis de façon explicite. Dans une première acception, le groupe restreint serait la simple réunion de trois à une quinzaine de joueurs. L'équipe renverrait plus à une organisation des tâches et des rôles dans une optique fonctionnelle en vue d'un objectif précis. De son côté, le collectif se parerait parallèlement à son organisation fonctionnelle d'une solidarité partagée à toute épreuve. Dans une équipe de sport collectif, les joueurs comme les entraîneurs sont confrontés à un apprentissage des phénomènes de groupe, le tout en relation avec le respect de règles éthiques qui sont le fondement de tous les jeux. Une équipe peut difficilement être appréhendée de façon ponctuelle, hors du contexte et hors de son évolution dans le temps. Les sports collectifs étant des sports d'équipe, les questions de tactique et d'intelligence collective sont fondamentales. Le résultat d'une rencontre ne dépend pas seulement de l'habileté des joueurs à agir sur la balle, mais aussi des choix tactiques des deux équipes dans un contexte d'affrontement.

Dans les jeux de balle, le jeu en mouvement et l'écoulement du temps génèrent un enchaînement de configurations momentanées du jeu qui se succèdent de façon continue. La dynamique dans les groupes qui s'affrontent s'exprime par la circularité des problèmes posés et des problèmes à résoudre en vue d'atteindre à la fois l'objectif qui finalise l'action des joueurs partenaires, tout en faisant obstacle aux entreprises des joueurs adversaires. Les multiples possibilités de placement, de déplacement, replacement donnent au jeu un caractère d'incertitude, renforcé par le fait que l'initiative change de camp avec le gain ou la perte de la balle. A propos des sports collectifs, le vrai miracle reste la naissance d'une grande équipe et tout ce qui peut y contribuer mérite considération. Quoi qu'il advienne, l'entraîneur constituera toujours, avec son groupe, ce duo où la différence est considérable lorsque c'est un grand meneur d'hommes qui le dirige, ou lorsque c'est un chef médiocre. Toutefois, d'un collectif de joueurs à une équipe, un long chemin est à parcourir. Pour mieux comprendre les enjeux d'une telle affirmation, nous allons envisager dans ce texte, de regrouper et développer des éléments théoriques que nous avons déjà abordés (Gréhaigne, 1997 ; Gréhaigne, Billard \& Laroche, 1999 ; Gréhaigne, 2009). Il s'agira de préciser et d'approfondir 
quelques conceptions à propos des étapes et des incontournables en vue de passer d'un groupe à une équipe de sport collectif : c'est-à-dire comprendre une histoire, des engagements dans le temps.

Qu'est-ce qu'une équipe ? Quelles modalités de constitution des équipes ?

Pour tenter de répondre à ces questions, et après une première partie relatant quelques éléments de la littérature concernant les groupes et leur fonctionnement, nous analyserons différentes caractéristiques des formes de groupement. Dans la discussion, nous envisagerons les leçons que nous pouvons tirer de cet ensemble pour mieux comprendre le fonctionnement des équipes de sport collectif.

\section{Le groupe et les sports collectifs dans la tradition francophone.}

Le mot équipe remonterait au moyen âge et proviendrait, du mot esquif (en allemand schiff, en anglais ship), terme qui signifie petite embarcation. Puis, par extension, le mot représente leurs occupants dans leur activité collective que l'on retrouve par extension dans le terme équipage (Bernard, 1963). Objectivement, un groupe relativement restreint est constitué d'individus unis pour réaliser un objectif commun. Une équipe, en effet, comprend rarement plus d'une vingtaine de membres. De nombreuses études ont été menées concernant la dynamique de l'équipe (sa genèse, sa cohésion, son climat, son réseau de communications, sa préparation psychologique, ses styles de « leadership »). Devant l'abondance de la littérature, nous ne retiendrons, à titre d'exemple, que quelques auteurs et le thème de leur travail : Rioux et Chappuis (1976), Gilbert, \& Trudel, (2012) ; Buton, Fontayne, \& Heuzé (2006) sur la cohésion de l'équipe ; Carron (1982, 1990) à propos de la taille des groupes sportifs ; Rey (2001), Amado \& Fustier, (2012) sur l'étude des effets sur l'intervention auprès d'équipes. Ce sont des auteurs qui ont également beaucoup contribué à l'étude des groupes sportifs. Les méthodes utilisées sont les sociogrammes, les questionnaires et, plus récemment, les tests projectifs spécialisés. Mais ce type de travail reste trop particulier pour prétendre être une aide efficace pour les formateurs.

Mérand (1977) présente les jeux sportifs collectifs comme « une dimension spécifique, une modalité originale d'exploitation de la dynamique des groupes restreints poursuivant un objectif commun» (p. 12). Les joueurs doivent saisir comment s'organisent dialectiquement les actions individuelles en vue de réaliser une action commune. Il faut, dans un même acte, empêcher l'adversaire de réussir un but ou un panier en protégeant sa cible mais aussi en tentant de se procurer l'occasion de marquer dans un proche avenir. Enfin, le rapport des forces en présence ou « rapports d'opposition » (Deleplace, 1979 ; Gréhaigne, 1989 ; Gréhaigne, Godbout \& Bouthier, 1999) entre deux équipes, dans un contexte d'apprentissage, peut être défini par des liens antagonistes existant entre plusieurs joueurs ou groupes de joueurs opposés du fait de l'application de certaines règles du jeu qui déterminent un mode d'interaction. Avec l'observation du jeu, cela devrait constituer la base 
d'analyse incontournable pour le système enseignement / apprentissage dans les sports où des équipes s'affrontent.

\section{Le coach : une approche américaine.}

Dans l'imaginaire collectif du sport, être coach c'est avoir la passion du management pour les sportifs que l'on encadre, avec comme crédo : tous ont besoin d'un environnement performant et d'être impliqués en vue de prendre le projet à leur compte. Le monde du sport, c'est un monde d'urgence, l'urgence de la performance. Tout compte fait, la meilleure façon de l'atteindre, c'est de tout contrôler, de tout maîtriser, de tout diriger et de n'emmener avec soi que des gens qui sont dans sa propre ligne. Lorsque vous dirigez un groupe de manière autoritaire, vous avez des joueurs, temporairement obéissants, mais certains peuvent aussi être résistants. Les entraîneurs sont responsables de la création d'un environnement sûr et stimulant et doivent fournir des expériences enrichissantes pour tous leurs joueurs. Ces environnements sont souvent appelés culture d'équipe et il existe de nombreuses techniques que les entraîneurs peuvent utiliser pour la construire.

La réflexion aide à se rappeler pourquoi on est entraîneur et aide à être fidèle à ses croyances. Qu'est-ce qui guide vos actions ? Si vous ne croyez pas en ce que vous faites ou essayez d'être quelqu'un que vous n'êtes pas, il sera difficile de fixer des normes pour votre équipe et encore plus difficile pour établir une crédibilité lors du renforcement de ces normes. Plus vous prenez de l'âge, plus l'écart de génération entre vous, vos assistants et vos joueurs devient grand. Continuez de trouver des façons de rester pertinent aux yeux de ceux que vous dirigez. Plus le lien personnel que vous partagez avec chaque joueur est fort, plus il sera facile pour mettre en œuvre la construction d'équipe avec la perspective de culture que vous recherchez. Enfin, la longévité d'un coach dépend de sa volonté d'apprendre de façon continue et de s'améliorer en tant que leader sportif (Mallett, Rynne, \& Dickens, 2013). Même si la méthode que vous avez utilisée pour construire la culture d'équipe dans le passé a réussi, vous pouvez continuellement découvrir d'autres approches ou de légères modifications qui peuvent faire toute la différence.

On peut affirmer sans crainte que c'est une conception très magistro-centrée avec un style de leadership plutôt directif où les rôles et les tâches sont définis par le coach qui supervise étroitement leur exécution. La position de principe, c'est une main ferme pour prendre des décisions rapidement, notamment en cas d'urgence à gérer. Van Mullem, \& Showalter (2018) ajoutent que les très grands entraîneurs maîtrisent tellement l'environnement qu'ils peuvent transcender le jeu et aussi avoir une influence durable sur chaque membre de l'équipe, même après que leur collaboration ait pris fin. On n'est pas loin du gourou qui se sert de son influence et de manipulations pour « asservir » des personnes ! 


\section{Des données pour l'analyse d'une équipe}

La vie d'un groupe paraît, au premier abord, limpide et facilement compréhensible. En réalité, il n'en est rien. Dès que l'on regarde de plus près, les actions collectives sont complexes, multiformes voire parfois mystérieuses en relation avec un sentiment d'appartenance plus ou moins affirmé. Pourtant, peu échappe à l'influence du groupe auquel il appartient et, fréquemment, on ne peut pas envisager d'analyser les comportements, les croyances et les attitudes d'un joueur si l'on ignore les normes et les valeurs du groupe auxquelles il a adhéré.

Aussi, un des grands thèmes de la didactique des sports collectifs concerne, bien entendu, le groupe de joueurs qui constitue une équipe. Dans la définition des jeux sportifs collectifs proposée par Gréhaigne (1992), le rapport de forces est caractérisé par « un groupe affrontant un autre groupe » (p. 22). Anzieu et Martin (1968) puis Carron (1988) définissent « un groupe primaire restreint comme étant la réunion de trois à quinze personnes ». Avant, on a un couple puis un rassemblement, le tout avec des fonctionnements fort différents.

L'équipe comme une organisation sociale.

Dans un groupe qui joue, il ne faut pas négliger les aspects socio-cognitifs du jeu sportif collectif. Ces derniers supposent une interaction sociale parce qu'elle se produit dans un système social qui la rend possible, la situe et lui donne une certaine historicité (Rey, 2001). En mettant en perspective l'inévitable insertion des conduites du joueur dans ce qu'on appelle la symbolique sociale, il s'agit de tenir compte de conduites en jeu qui sont la conséquence d'autres déterminants et qui ne trouvent pas forcément un ancrage dans les caractéristiques intrinsèques de la situation de jeu.

La cohésion d'un groupe ne forme habituellement pas un tout homogène, monolithique. L'équipe au contraire est organisée, c'est-à-dire comporte un minimum de différenciation entre les individus qui la composent. Cette différenciation n'est pas inhérente aux individus et par conséquent accidentelle (c'est-à-dire due aux hasards de la naissance, aux différences constitutionnelles ou aux variétés du tempérament et du caractère...). Elle est au contraire inventée et nécessaire : c'est une différenciation fonctionnelle. L'organisation est, en effet, primitivement répartition de fonctions, c'est-à-dire distribution de tâches aux individus qui se sont groupés afin de mieux assurer la coordination de leurs actions en vue de la réalisation de l'objectif commun. La coopération entre les joueurs nécessite à la fois des interactions et une confiance partagée, celles-ci se construisant progressivement au gré de la vie de l'équipe.

Stabilité du groupe, permanence, durée et histoire du groupe.

La répartition des tâches et des fonctions est essentiellement commandée par un objectif commun. Comme le dit Sartre (1960), «le mouvement organisateur décide du rapport entre les hommes en 
fonction de la relation fondamentale du groupe avec la chose » (p. 460). Le groupe n'agit sur son objectif que dans la mesure où il agit sur lui-même. Encore faut-il cependant que cette différenciation soit compatible avec l'existence même du groupe, c'est-à-dire qu'elle ne brise pas son unité. Cette condition est réalisée par l'engagement initial, devenu permanent des individus qui sont entrés dans l'équipe. Il doit y avoir un acte d'adhésion par lequel chacun lie sa liberté à la liberté des autres, et cela par le truchement matériel d'une signature, d'une parole ou d'un geste. Cet engagement constitue la garantie contre les dangers d'une dissolution consécutive à l'individualisation et à la relative autonomie créée par la répartition des tâches. Ainsi, le joueur vedette qui confisque à son profit le succès du travail de l'équipe peut faire éclater cette équipe et crée autant de tentations pour les autres de l'imiter en surévaluant le rôle qui leur est dévolu. Par conséquent, la structure différenciée de l'équipe est immédiatement intelligible et ne pose, du moins à ce niveau, aucun problème.

Par contre, ce qui reste difficilement accessible et constitue le problème fondamental de l'équipe, c'est de savoir comment interpréter l'action organisée de l'équipe elle-même sur l'objectif visé en commun, c'est-à-dire comprendre le rapport de l'action du groupe sur lui-même par l'organisation avec l'action de chacun de ses membres sur l'objet commun. Concrètement, dans le cas d'une équipe sportive comme celle de football, cela revient à comprendre le rapport entre la structure différenciée de l'équipe (répartition des postes : gardien de but, arrières, milieux et avants, sans compter la distribution interne à ce niveau du centre à la périphérie) avec 1'action de tous les joueurs à la conquête du but adverse. Ce problème théorique se traduit sur le plan pratique par cette question qui se pose à tous les entraîneurs : comment organiser mon équipe pour obtenir le maximum d'efficacité des actions individuelles et collectives dans les situations indéfiniment variables ? Cela constitue le problème premier de toute conception de la dynamique de l'équipe sportive.

\section{Le groupe organisé}

Dans une perspective dialectique sartrienne, le groupe se définira toujours par ses actions et non comme un super organisme ou comme une conscience collective. Il faut bien se rappeler que cette autonomie n'est qu'apparente, car la réalité du groupe n'est pas, en fait, indépendante de l'action de ses membres. En tant qu'entité organisée, l'équipe est un groupe qui, par une action intérieure, définit elle-même ses structures.

Ainsi, il s'agit de déterminer ou de fixer à l'avance, cet avenir en inventant une conduite qui engage le groupe à demeurer permanent avec un projet. Cette conduite est un engagement de chacun devant tout le monde. Grâce à cette structure nouvelle, le groupe peut affronter les risques présentés par une diversité de tâches, c'est-à-dire par une différenciation de ses membres. En conséquence, le groupe statutaire permet « le groupe organisé », qui attribue du pouvoir à chaque membre sur les 
autres par la répartition ou parfois l'assignation de «fonctions ». La fonction est, en effet, une détermination de l'action individuelle : chaque individu appartient au groupe en tant qu'il exécute une certaine tâche et seulement celle-là. Accomplir telle tâche assignée par la fonction est alors, en vertu du serment, en même temps un droit et un devoir puisque la tâche marque le droit de chacun sur tous et de tous sur chacun.

Tel est le processus qui préside à la constitution de toute équipe et d'une équipe sportive en particulier. En effet, dans une équipe de football, la fonction de gardien de but ou d'attaquant est, en quelque sorte, une manière de limiter à l'avance la liberté du jeune joueur qui débute, une prédétermination de son comportement. C'est la fonction qui permet son recrutement : la fonction le sélectionne en tenant compte de ses qualités physiques aussi bien que psychologiques (motivation, engagement, intelligence tactique ...). Mais, dans la mesure où elle crée cette détermination dans la liberté du joueur, la fonction crée en même temps chez celui-ci un pouvoir. Le pouvoir donné à ce joueur d'exiger qu'on lui facilite l'exercice de sa fonction et pour cela qu'on l'entraîne : L'équipe a le devoir de l'élever jusqu'au niveau (physique et tactique) où il sera à même de produire les actes que le groupe exige (Sartre, 1960). C'est en tant qu'individu commun, c'est-à-dire ayant consenti à soumettre son action à l'action collective, que ses droits lui appartiennent. À ce niveau, il n'y a aucune différence entre droit et devoir. C'est cette structure juridique et dialectique (réciprocité des droits et des devoirs) qui définit l'existence de l'équipe sportive.

L'interprétation dialectique garde donc toute sa valeur. Elle seule semble pouvoir rendre compte et du mouvement constituant l'équipe et de l'intégration incessante des actions individuelles à ce mouvement. L'élément fondateur est constitué par la volonté délibérée de situer le problème de la dynamique des groupes et tout particulièrement de l'équipe dans le temps, dans son devenir plus que dans l'espace. En cela, l'interprétation dialectique de Sartre s'oppose à celle de la Gestalt-théorie, en particulier à Lewin qui tend à projeter les phénomènes sociaux dans des « champs » conçus et représentés spatialement, créant ainsi une psychosociologie «topologique ». La réalité du groupe est, au contraire pour Sartre, son histoire : histoire qui n'est pas un destin, c'est-à-dire qui n'est pas réductible à un déterminisme des propriétés structurelles du groupe comme gestalt ou comme une totalité achevée étudiée du dehors par le sociologue. Sur le plan pratique, cette manière de voir revient à éviter de traiter le joueur comme un objet, un sujet ou une mécanique.

\section{L'équipe de sport collectif}

Avec cette notion de temps au cœur de l'analyse, l'équipe est caractérisée par un paradoxe fondamental : elle possède un passé, un présent, et peut être un futur. Si l'on examine attentivement la totalité des dimensions du temps, on se rend compte que le passé n'est plus, le futur n'est pas encore et que le présent est sans cesse fluctuant. Il convient donc d'envisager l'équipe dans son 
processus de constitution, d'évolution et d'organisation mais aussi dans ses conflits, ses tensions, ses réseaux, en résumé dans son processus ou sa tendance à redevenir un ensemble d'individus. Pour la pérennité du groupe, le bon entraîneur saura réintégrer ces conflits plutôt que les refouler : il devra agir de façon à les transformer en une occasion de dépassement vers une nouvelle formule de cohésion plutôt que s'efforcer à récupérer une unité primitive désormais dépassée. D'autre part, cette perspective historique nous invite à ne pas juger un match en soi, isolé de son contexte temporel. Pour le spectateur aussi bien que pour l'entraîneur, il conviendra parallèlement de prendre conscience qu'ils participent à l'histoire de l'équipe qu'ils voient, font jouer ou aident à jouer. Ils contribuent à leur construction par leurs propres attitudes, leurs actions ou leurs réactions.

Néanmoins, le type d'activité sportive semble avoir une forte incidence sur les stratégies mobilisées par les entraîneurs. Ces derniers activent des ensembles cohérents de stratégies selon le groupe sportif qu'ils dirigent. Par exemple, les entraîneurs de rugby interviennent plutôt sur le pôle relationnel et affectif. Ils s'appuient sur le capitaine de l'équipe pour faire passer leurs décisions. Ils engagent la discussion avec l'équipe tout en valorisant les valeurs fédérant le groupe. Par contre, les entraîneurs d'handball montrent leur volonté de marquer leur autorité. Ils souhaitent la confiance de l'équipe qu'ils sont prêts à impressionner, à défaut de sanctionner (Rey, 2001).

Tout cet ensemble constitue, bien entendu, un tissu complexe de relations sur lesquelles nous allons revenir en puisant nos exemples dans les jeux de balle.

\section{Réseau de compétences}

Dans le jeu, les positions successives et les mouvements respectifs des joueurs et du ballon sont pris en compte pour caractériser le rapport d'opposition. En fonction d'un rapport de forces donné, s'instaurent, dans chaque équipe, une distribution des tâches, des conflits ou une répartition tacite des rôles et des fonctions que nous appelons réseau de compétences. Celui-ci est constitué par des conduites et des comportements qui peuvent être identifiés chez les élèves ou les joueurs, à l'intérieur d'une équipe, en fonction de la façon dont est vécu le rapport de forces. L'ensemble de ces conduites varie suivant les joueurs, les moments, les facteurs extérieurs et les sports collectifs (Gréhaigne, Billard, \& Laroche, 1999). Dans le jeu, la notion de rôle (conduite qui exprime ce que le joueur pense avoir à faire en rapport avec la façon dont il vit le rapport de forces, les relations avec ses partenaires dans l'équipe et comment il gère ses ressources dans ce système de contraintes) est essentielle à l'analyse de ce type de comportements. La fonction dans le groupe que le joueur choisit ou qui lui est assignée par l'entraîneur ou par le groupe est un autre révélateur de sa position dans la dynamique de l'équipe. À l'interface de la logique du sujet, de l'histoire du groupe et de la logique interne du sport considéré, la place du joueur dans ce réseau de compétences constitue souvent un révélateur fiable des rapports réciproques entre ce joueur, certains autres, l'équipe dans 
l'ensemble et les adversaires. Parfois, cela peut même prendre la forme d'un conflit aigu entre des joueurs qui sont pourtant partenaires! Cet état de fait confirme pleinement que les rapports «individu / groupe» et «groupe / individu» constituent bien un des points clés de la compréhension de l'organisation d'une équipe.

L'équipe et le joueur.

Dans sa classification des activités sportives, Jeu (1977) range les sports collectifs dans la catégorie compétition qu'il caractérise ainsi «de l'égalité des chances au départ à l'inégalité du résultat» (p. 178). L'analyse d'une rencontre et de son résultat doit coordonner les aspects liés à l'organisation du jeu en relation avec les décisions individuelles prises par les joueurs. En effet, pour des joueurs, une même adaptation à un affrontement donné dépendra de la combinatoire de différents facteurs, donc de niveau d'organisation, où la hiérarchie entre ses ressources et les contraintes du jeu pourra être fort différente pour un résultat apparemment semblable. Le joueur de sport collectif est aussi le résultat, à cet instant précis, d'une relation complexe entre le poste qu'il occupe, ses qualités physiques, ses ressources motivationnelles et ses savoirs. Cet état d'équilibre est précaire et sa principale caractéristique est la réversibilité. En fonction d'un rapport de forces vécu défavorablement, un joueur peut revenir à des conduites inférieures à son niveau théorique relevant d'une adaptation à l'affrontement. Cette approche devrait proposer une autre conception des conduites communes à tous les individus comme une conception plurielle prenant en compte le groupe comme fait premier et signifiant les différences entre les procédures adaptatives mises en œuvre, dans la même situation par des individus différents ou par le même individu en des moments successifs. Dans ce cas, les joueurs ont bien compris que s'associer n'est pas forcément passer ses vacances ensemble mais que cela constitue une démarche cognitive pour accéder à un autre niveau de résultat et ainsi profiter de ce que ce résultat procure à titre individuel. C'est donc une démarche intelligente et fonctionnelle que l'on demande aux joueurs d'adopter. Comme dans l'approche dialectique de la dynamique de l'équipe (Sartre, 1960), quand ce ne sont pas les bons sentiments d'amitié qui gouvernent les relations, on n'accepte pas ce qui crée du désordre. Il y a donc une espèce de régulation par rapport à l'engagement, l'investissement et la motivation de chacun qui fait que le groupe et chacun contrôle tout le monde. Face à ce problème, Rey (1990) se réfère à une psychologie sociale cognitive qui tente de préciser le concept de cognition sociale. Il s'agit ici pour le joueur de repérer la valeur sociale de la situation de jeu et de traiter avant tout son utilité sociale. Dans cette perspective, l'interaction tactique devient un espace social, non seulement parce qu'elle implique la présence d'autres joueurs, mais aussi parce qu'elle peut se définir comme un espace de comparaison et de distinction sociales. Ce singularisme définit une nouvelle relation entre le joueur et l'équipe, où les enjeux identitaires se substitueraient en partie aux enjeux stratégiques et 
tactiques. Les expériences individuelles deviennent l'horizon d'attentes de la perception du jeu car c'est d'abord en référence à celles-ci que le joueur agit. Un joueur acteur social, ancré dans une situation sociale singulière, datée et située, met en place des comportements particuliers. Dans cette situation sociale, les relations entraîneurs / joueurs transforment également le sens de l'interaction tactique.

\section{Le vestiaire}

Une équipe est une communauté qui fonctionne en symbiose entre le coach ou l'entraîneur quand tout va bien. L'entraîneur peut considérer que ces discussions dans le vestiaire sont indispensables et constituent une sorte de soupape de sûreté pour la vie du groupe. Le vestiaire proprement dit est constitué par l'ensemble des joueurs et est fondé sur une hiérarchie des compétences, les influences et les réseaux amicaux. Le vestiaire peut être le lieu de mise au point, de discussion voire de mise en cause des responsables. Ce genre de problème peut très vite déraper et, à ce moment-là, l'entraîneur se retrouve en première ligne et mis en cause. Si vous dirigez un groupe de manière autoritaire, vous avez des joueurs obéissants mais certains aussi résistants car il y a toujours des leaders dans un groupe. S'il est leader, le joueur n'existe que par sa capacité à résister. Et si les autres le suivent, c'est qu'ils lui reconnaissent cette capacité à résister et peut-être, par-là, défendre leur place dans le réseau de compétences. Mais qu'est-ce qui fait que vous allez gagner un match très équilibré entre deux équipes équivalentes ? Qu'est-ce qui fait que c'est toujours la même équipe qui gagne d'un seul but et souvent les mêmes qui perdent d'un but ? L'équipe qui gagne a, certes, l'expérience qui fait fréquemment défaut au groupe trop jeunes. Mais on peut penser que c'est également la capacité des joueurs à se faire confiance, à être complices et à pouvoir s'associer précisément au moment où les joueurs de l'équipe adverse, angoissés par le risque de l'échec sont, déjà, en train de penser à se justifier.

Pour un entraîneur, la compétition haut niveau est trop difficile pour qu'on puisse entreprendre ce travail prenant et difficile tout seul (Amado, 2012). Alors, pourquoi ne pas partager cette charge ensemble avec les joueurs ? Dans le cas d'un conflit entre le vestiaire et l'entraîneur, il est très rare que celui-ci en sorte sans dommage. En effet, le jour où les joueurs ne reconnaissent plus au coach la capacité à résoudre les problèmes, ils vont essayer de les résoudre par eux-mêmes et risquent alors de créer ces fractures irréversibles tout en ayant mis en évidence que l'entraîneur ne fait plus consensus autour de lui. Celui-ci ne plus être la personne qui exerce une autorité ou une influence déterminante parce qu'il n'a pas assumé son rôle qui est celui de la prise de décision et parfois de la prise de sanction. Cela peut être douloureux, mais il faut comprendre que cet état de fait est nécessaire pour maintenir ou rétablir l'équilibre du groupe. Le plus souvent, l'entraîneur est remercié par les dirigeants en espérant que ce fameux choc psychologique redonne de l'énergie à 
une équipe souvent à bout de souffle. Le coach est bien considéré comme un fusible.

\section{Conclusion.}

Ce large tour d'horizon était destiné à mieux comprendre l'histoire d'un groupe, d'une équipe. Mais, en sport collectif, ce ne sont pas seulement les interactions entre individus qui structurent un groupe. C'est aussi l'interaction avec d'autres groupes en relation avec les moments de stress collectif dû à cet affrontement. Ici, ce qui émerge ce ne sont pas les préférences individuelles mais les contraintes d'un niveau supérieur d'interaction qui renvoient donc à une réalité à la fois subie et construite. En définitive, il nous a semblé intéressant de montrer dans ce texte ce qui oppose les aspects dialectiques du phénomène «groupe », avec leur caractère parfois destructeur et une conception des phénomènes organisationnels fondés sur la libre circulation de l'information, la coopération et l'adaptation de joueurs libres et autonomes ...

Dans les sports collectifs, on constate souvent que les meilleures équipes ne sont pas forcément celles composées des meilleurs joueurs pris un par un à chaque poste. Une équipe n'est donc pas tant composée des meilleurs joueurs mais de ceux qui ont appris à jouer ensemble, de ceux qui sont complémentaires et dont la contribution au groupe est la meilleure possible, même dans les tâches les plus obscures. Un groupe, quel qu'il soit, ne vaut en définitive que par son maillon le plus faible. Qu'il s'agisse d'une cordée d'alpinistes ou d'une équipe de sport collectif, la force ou le niveau du plus faible du groupe conditionne, aussi, l'avancée du dit groupe.

Enfin, dernière remarque, tout au long de cet exposé, nous avons peu évoqué un sujet pourtant fondamental dans la vie d'un groupe : les émotions. Oui, le stress, la peur, la jubilation, l'anxiété et la joie font partie de ces moments forts qui ponctuent la vie du groupe. Ces éléments sont le moteur, l'énergie d'une équipe qui en a besoin pour survivre tout en relevant d'une histoire à construire et à capitaliser.

\section{Bibliographie}

Anzieu, D., \& Martin, J.Y. (1968). La dynamique des groupes restreints. Paris : PUF.

Amado, G. (2012). Claude Onesta : Fabriquer une équipe double championne olympique. Nouvelle revue de psychosociologie, 14, (2), 191-204.

Amado, G., \& Fustier, P. (2012). Introduction faire équipe. Nouvelle revue de psychosociologie, 14, (2), 7-10.

Bernard, M. (1963). Une interprétation dialectique de la dynamique de l'équipe. E.P.S., 62, 7-11. 
Buton, F., Fontayne, P., \& Heuzé J.P. (2006). La cohésion des groupes sportifs : évolutions conceptuelles, mesures et relations avec la performance. Science et motricité, 59 (3), 0945.

Carron A.V. (1982). Cohesiveness in sport groups. Journal ofSport Psychology, 4/2. 123-138.

Carron A.V. (1990). Group size and physical activity : social psychological and performance consequences. International Journal of Sport Psychology, 21, 286-304.

Deleplace, R. (1979). Rugby de mouvement - Rugby total. Paris : Éducation Physique et Sport.

Gilbert, W., \& Trudel, P. (2012). The role of deliberate practice in becoming an expert coach: Part 1-Defining coaching expertise. Olympic Coach Magazine, 23(3), 19-24.

Gréhaigne, J.F. (1989). Football de mouvement. Vers une approche systémique du jeu. Thèse (nouveau régime). Université de Bourgogne.

Gréhaigne, J-F. (1997). Les formes de groupement en sports collectifs : Des aspects contradictoires. Revue EP\&S, 260, 35-36.

Gréhaigne, J.-F. (2009). D'un collectif de joueurs à une équipe : une histoire de temps. In J.-F. Gréhaigne (Ed.) Autour du temps. Espaces, apprentissages, projets dans les sports collectifs (pp. 75-84). Besançon : Presses de l’Université de Franche-Comté.

Gréhaigne, J.F., Billard, M., \& Laroche, J.Y. (1999). L'enseignement des jeux sportifs collectifs à l'école. Conception, construction, évaluation. Bruxelles : De Boeck.

Gréhaigne, J.-F. , Godbout, P., \& Bouthier, D. (1999). The foundations of tactics and strategy in team sports. Journal of Teaching in Physical Education, 18, 159-174.

Jeu, B. (1977). Le sport, l'émotion, l'espace. Paris : Vigot.

Mallett, C., Rynne, S., \& Dickens, S. (2013). Developing high performance coaching cra through work and study. In P. Potrac, W. Gilbert, \& J. Denison (Eds.), Routledge Handbook of Sports Coaching (463-475). New York, NY: Routledge.

Mérand, R. (1977). Considérations sur une problématique de rénovation des contenus de l'Education Physique en rapport avec les activités sportives contemporaines. In CPS de la FSGT (Ed.) L'éducateur face à la haute performance olympique (pp.05-27). Paris : Sport et Plein air.

Rey, J.P. (1990). Etude des effets de quelques comportements du manager sur les conduites de jeunes joueurs et joueuses de handball. Thèse (nouveau régime). Université de ClermontFerrand.

Rey, J.P. (2001). Le groupe. Paris : Revue EPS.

Rioux, R., \& Chappuis, G. (1976). La cohésion de l'équipe. Paris : Vrin.

Sartre, J.P. (1960). Critique de la raison dialectique. Paris : Gallimard. 
Van Mullem, P., \& Showalter D. (2018). Building Team Culture: "Mind Candy" for Coaches. Physical \& Health Education America News. February 2018. 